\title{
New algebraic aspects of perturbative and non-perturbative Quantum Field Theory
}

\author{
Christoph Bergbauer ${ }^{1,4}$ and Dirk Kreimer ${ }^{2,3}$ \\ ${ }^{1}$ Freie Universität Berlin, Institut für Mathematik II \\ Arnimallee 3, 14195 Berlin, Germany \\ ${ }^{2} \mathrm{CNRS}$ at Institut des Hautes Etudes Scientifiques \\ 35 route de Chartres, 91440 Bures-sur-Yvette, France \\ ${ }^{3}$ Boston University, Center for Mathematical Physics \\ 111 Cummington Street, Boston, MA 02215, USA \\ ${ }^{4}$ Erwin-Schrödinger-Institut \\ Boltzmanngasse 9, 1090 Wien, Austria \\ bergbau@math.fu-berlin.de, kreimer@ihes.fr
}

April 1, 2007

\begin{abstract}
In this expository article we review recent advances in our understanding of the combinatorial and algebraic structure of perturbation theory in terms of Feynman graphs, and Dyson-Schwinger equations. Starting from Lie and Hopf algebras of Feynman graphs, perturbative renormalization is rephrased algebraically. The Hochschild cohomology of these Hopf algebras leads the way to Slavnov-Taylor identities and Dyson-Schwinger equations. We discuss recent progress in solving simple Dyson-Schwinger equations in the high energy sector using the algebraic machinery. Finally there is a short account on a relation to algebraic geometry and number theory: understanding Feynman integrals as periods of mixed (Tate) motives.
\end{abstract}

\section{Introduction}

As elements of perturbative expansions of Quantum field theories, Feynman graphs have been playing and still play a key role both for our conceptual understanding and for state-of-the-art computations in particle physics. This 
article is concerned with several aspects of Feynman graphs: First, the combinatorics of perturbative renormalization give rise to Hopf algebras of rooted trees and Feynman graphs. These Hopf algebras come with a cohomology theory and structure maps that help understand important physical notions, such as locality of counterterms, the beta function, certain symmetries, or DysonSchwinger equations from a unified mathematical point of view. This point of view is about self-similarity and recursion. The atomic (primitive) elements in this combinatorial approach are divergent graphs without subdivergences. They must be studied by additional means, be it analytic methods or algebraic geometry and number theory, and this is a significantly more difficult task. However, the Hopf algebra structure of graphs for renormalization is in this sense a substructure of the Hopf algebra structure underlying the relative cohomology of graph hypersurfaces needed to understand the number-theoretic properties of field theory amplitudes [6, [5].

\section{Lie and Hopf algebras of Feynman graphs}

Given a Feynman graph $\Gamma$ with several divergent subgraphs, the Bogoliubov recursion and Zimmermann's forest formula tell how $\Gamma$ must be renormalized in order to obtain a finite conceptual result, using only local counterterms. This has an analytic (regularization/extension of distributions) and a combinatorial aspect. The basic combinatorial question of perturbative renormalization is to find a good model which describes disentanglement of graphs into subdivergent pieces, or dually insertion of divergent pieces one into each other, from the point of view of renormalized Feynman rules. It has been known now for several years that commutative Hopf algebras and (dual) Lie algebras provide such a framework [26, 14, 15] with many ramifications in pure mathematics. From the physical side, it is important to know that, for example, recovering aspects of gauge/BRST symmetry [39, 37, 30, 38, and the transition to nonperturbative equations of motion [12, 28, 29, 36, 3, 35, 32, 34, 4, are conveniently possible in this framework, as will be discussed in subsequent sections.

In order to introduce these Lie and Hopf algebras, let us now fix a renormalizable quantum field theory (in the sense of perturbation theory), given by a local Lagrangian. A convenient first example is massless $\phi^{3}$ theory in 6 dimensions. We look at its perturbative expansion in terms of 1PI Feynman graphs. Each 1PI graph $\Gamma$ comes with two integers, $|\Gamma|=\operatorname{rank} H_{1}(\Gamma)$, its number of loops, and $\operatorname{sdd}(\Gamma)$, its superficial degree of divergence. As usual, vacuum and tadpole graphs need not be considered, and the only remaining superficial divergent graphs have exactly two or three external edges, a feature of renormalizability. Graphs without subdivergences are called primitive. Here are two examples.

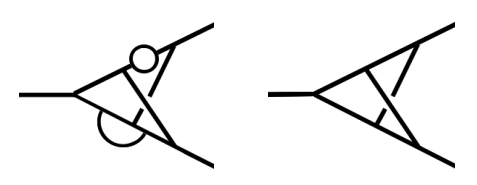


Both are superficially divergent as they have three external edges. The first one has two subdivergences, the second one is primitive. Note that there are infinitely many primitive graphs with three external edges. In particular, for every $n \in \mathbb{N}$ one finds a primitive $\Gamma$ such that $|\Gamma|=n$.

Let now $L$ be the $\mathbb{Q}$-vector space generated by all the superficially divergent (sdd $\geq 0$ ) 1PI graphs of our theory, graded by the number of loops $|\cdot|$. There is an operation on $L$ given by insertion of graphs into each other: Let $\gamma_{1}, \gamma_{2}$ be two generators of $L$. Then

$$
\gamma_{1} \star \gamma_{2}:=\sum_{\Gamma} n\left(\gamma_{1}, \gamma_{2}, \Gamma\right)
$$

where $n\left(\gamma_{1}, \gamma_{2}, \Gamma\right)$ is the number of times that $\gamma_{1}$ shows up as a subgraph of $\Gamma$ and $\Gamma / \gamma_{1} \cong \gamma_{2}$. Here are two examples:

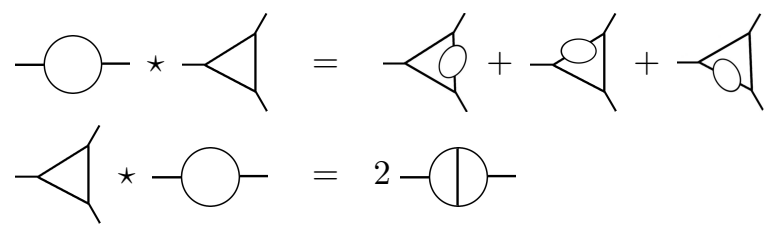

This definition is extended bilinearly onto all of $L$. Note that $\star$ respects the grading as $\left|\gamma_{1} \star \gamma_{2}\right|=\left|\gamma_{1}\right|+\left|\gamma_{2}\right|$. The operation $\star$ is not in general associative. Indeed, it is pre-Lie [14, 17]:

$$
\left(\gamma_{1} \star \gamma_{2}\right) \star \gamma_{3}-\gamma_{1} \star\left(\gamma_{2} \star \gamma_{3}\right)=\left(\gamma_{1} \star \gamma_{3}\right) \star \gamma_{2}-\gamma_{1} \star\left(\gamma_{3} \star \gamma_{3}\right) .
$$

To see that (10) holds observe that on both sides nested insertions cancel. What remains are disjoint insertions of $\gamma_{2}$ and $\gamma_{3}$ into $\gamma_{1}$ which do obviously not depend on the order of $\gamma_{2}$ and $\gamma_{3}$. One defines a Lie bracket on $L$ :

$$
\left[\gamma_{1}, \gamma_{2}\right]:=\gamma_{1} \star \gamma_{2}-\gamma_{2} \star \gamma_{1} .
$$

The Jacobi identity for $[\cdot, \cdot]$ is satisfied as a consequence of the pre-Lie property (11) of $\star$. This makes $L$ a graded Lie algebra. The bracket is defined by mutual insertions of graphs. As usual, $\mathcal{U}(L)$, the universal envelopping algebra of $L$ is a cocommutative Hopf algebra. Its graded dual, in the sense of Milnor-Moore, is therefore a commutative Hopf algebra $\mathcal{H}$. As an algebra, $\mathcal{H}$ is free commutative, generated by the vector space $L$ and an adjoined unit $\mathbb{I}$. By duality, one expects the coproduct of $\mathcal{H}$ to disentangle its argument into subdivergent pieces. Indeed, one finds

$$
\Delta(\Gamma)=\mathbb{I} \otimes \Gamma+\Gamma \otimes \mathbb{I}+\sum_{\gamma \subsetneq \Gamma} \gamma \otimes \Gamma / \gamma .
$$

The relation $\gamma \subsetneq \Gamma$ refers to disjoint unions $\gamma$ of 1PI superficially divergent subgraphs of $\Gamma$. Disjoint unions of graphs are in turn identified with their product in $\mathcal{H}$. For example,

$$
\Delta(-)=\mathbb{I} \otimes-D-+-D-\otimes \mathbb{I}+2 \longleftrightarrow-O-
$$


The coproduct respects the grading by the loop number, as does the product (by definition). Therefore $\mathcal{H}=\bigoplus_{n=0}^{\infty} \mathcal{H}_{n}$ is a graded Hopf algebra. Since $\mathcal{H}_{0} \cong \mathbb{Q}$ it is connected. The counit $\epsilon$ vanishes on the subspace $\bigoplus_{n=1}^{\infty} \mathcal{H}_{n}$, called augmentation ideal, and $\epsilon(\mathbb{I})=1$. As usual, if $\Delta(x)=\mathbb{I} \otimes x+x \otimes \mathbb{I}$, the element $x$ is called primitive. The linear subspace of primitive elements is denoted Prim $\mathcal{H}$.

The interest in $\mathcal{H}$ and $L$ arises from the fact that the Bogoliubov recursion is essentially solved by the antipode of $\mathcal{H}$. In any connected graded bialgebra, the antipode $S$ is given by

$$
S(x)=-x-\sum S\left(x^{\prime}\right) x^{\prime \prime}, \quad x \notin \mathcal{H}_{0}
$$

in Sweedler's notation. Let now $V$ be a $\mathbb{C}$-algebra. The space of linear maps $\mathcal{L}_{\mathbb{Q}}(\mathcal{H}, V)$ is equipped with a convolution product $(f, g) \mapsto f * g=m_{V}(f \otimes$ $g) \Delta$ where $m_{V}$ is the product in $V$. Relevant examples for $V$ are suggested by regularization schemes such as the algebra $V=\mathbb{C}\left[\left[\epsilon, \epsilon^{-1}\right]\right.$ of Laurent series with finite pole part for dimensional regularization (space-time dimension $D=6+2 \epsilon$.) The (unrenormalized) Feynman rules provide then an algebra homomorphism $\phi: \mathcal{H} \rightarrow V$ mapping Feynman graphs to Feynman integrals in $6+2 \epsilon$ dimensions. On $V$ there is a linear endomorphism $R$ (renormalization scheme) defined, for example minimal subtraction $R\left(\epsilon^{n}\right)=0$ if $n \geq 0, R\left(\epsilon^{n}\right)=\epsilon^{n}$ if $n<0$. If $\Gamma$ is primitive, as defined above, then $\phi(\Gamma)$ has only a simple pole in $\epsilon$, hence $(1-R) \phi(\Gamma)$ is a good renormalized value for $\Gamma$. If $\Gamma$ does have subdivergences, the situation is more complicated. However, the map $S_{R}^{\phi}: \mathcal{H} \rightarrow V$

$$
S_{R}^{\phi}(\Gamma)=-R\left(\phi(\Gamma)-\sum S_{R}^{\phi}\left(\Gamma^{\prime}\right) \phi\left(\Gamma^{\prime \prime}\right)\right)
$$

provides the counterterm prescribed by the Bogoliubov recursion, and $\left(S_{R}^{\phi} *\right.$ $\phi)(\Gamma)$ yields the renormalized value of $\Gamma$. The map $S_{R}^{\phi}$ is a recursive deformation of $\phi \circ S$ by $R$, compare its definition with (3). These are results obtained by one of the authors in collaboration with Connes [26, 14, 15].

For $S_{R}^{\phi}$ to be an algebra homomorphism again, one requires $R$ to be a RotaBaxter operator, studied in a more general setting by Ebrahimi-Fard, Guo and one of the authors in 20, 22, 21. The Rota-Baxter property is at the algebraic origin of the Birkhoff decomposition introduced in [15, 16. In the presence of mass terms, or gauge symmetries etc. in the Lagrangian, $\phi, S_{R}^{\phi}$ and $S_{R}^{\phi} \star \phi$ may contribute to several form factors in the usual way. This can be resolved by considering a slight extension of the Hopf algebra containing projections onto single structure functions, as discussed for example in [15, 32]. For the case of gauge theories, a precise definition of the coefficients $n\left(\gamma_{1}, \gamma_{2}, \Gamma\right)$ is given in [30].

The Hopf algebra $\mathcal{H}$ arises from the simple insertion of graphs into each other in a completely canonical way. Indeed, the pre-Lie product determines the coproduct, and the coproduct determines the antipode. Like this, each quantum field theory gives rise to such a Hopf algebra $\mathcal{H}$ based on its 1PI graphs. It is 
no surprise then that there is an even more universal Hopf algebra behind all of them: The Hopf algebra $\mathcal{H}_{r t}$ of rooted trees [26, 14. In order to see this, imagine a purely nested situation of subdivergences like

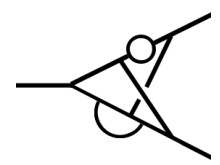

which can be represented by the rooted tree

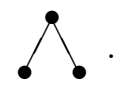

To account for each single graph of this kind, the tree's vertices should actually be labeled according to which primitive graph they correspond to (plus some gluing data) which we will suppress for the sake of simplicity. The coproduct on $\mathcal{H}_{r t}$ - corresponding to the one (2) of $\mathcal{H}$ - is

$$
\Delta(\tau)=\mathbb{I} \otimes \tau+\tau \otimes \mathbb{I}+\sum_{a d m . c} P_{c}(\tau) \otimes R_{c}(\tau)
$$

where the sum runs over all admissible cuts of the tree $\tau$. A cut of $\tau$ is a nonempty subset of its edges which are to be removed. A cut $c(\tau)$ is defined to be admissible, if for each leaf $l$ of $\tau$ at most one edge on the path from $l$ to the root is cut. The product of subtrees which fall down when those edges are removed is denoted $P_{c}(\tau)$. The part which remains connected with the root is denoted $R_{c}(\tau)$. Here is an example:

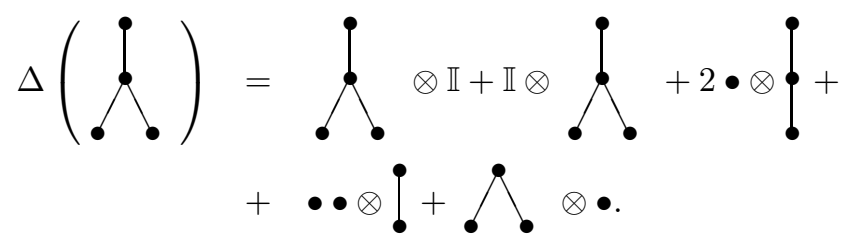

Compared to $\mathcal{H}_{r t}$, the advantage of $\mathcal{H}$ is however that overlapping divergences are resolved automatically. To achieve this in $\mathcal{H}_{r t}$ requires some care [27].

\section{From Hochschild cohomology to physics}

There is a natural cohomology theory on $\mathcal{H}$ and $\mathcal{H}_{r t}$ whose non-exact 1-cocycles play an important "operadic" role in the sense that they drive the recursion that define the full 1PI Green's functions in terms of primitve graphs. In order to introduce this cohomology theory, let $A$ be any bialgebra. We view $A$ as a bicomodule over itself with right coaction $(i d \otimes \epsilon) \Delta$. Then the Hochschild cohomology of $A$ (with respect to the coalgebra part) is defined as follows [14]: 
Linear maps $L: A \rightarrow A^{\otimes n}$ are considered as $n$-cochains. The operator $b$, defined as

$$
b L:=(i d \otimes L) \Delta+\sum_{i=1}^{n}(-1)^{i} \Delta_{i} L+(-1)^{n+1} L \otimes \mathbb{I}
$$

furnishes a codifferential: $b^{2}=0$. Here $\Delta$ denotes the coproduct of $A$ and $\Delta_{i}$ the coproduct applied to the $i$-th factor in $A^{\otimes n}$. The map $L \otimes \mathbb{I}$ is given by $x \mapsto L(x) \otimes \mathbb{I}$. Clearly this codifferential encodes only information about the coalgebra (as opposed to the algebra) part of $A$. The resulting cohomology is denoted $\mathrm{HH}_{\epsilon}^{\bullet}(A)$. For $n=1$, the cocycle condition $b L=0$ is simply

$$
\Delta L=(i d \otimes L) \Delta+L \otimes \mathbb{I}
$$

for $L$ a linear endomorphism of $A$. In the Hopf algebra $\mathcal{H}_{r t}$ of rooted trees (where things are often simpler), a 1-cocycle is quickly found: the grafting operator $B_{+}$, defined by

$$
\begin{aligned}
B_{+}(\mathbb{I}) & =\bullet \\
B_{+}\left(\tau_{1} \ldots \tau_{n}\right) & =\bigwedge_{\tau_{1} \ldots \tau_{n}} \text { for trees } \tau_{i}
\end{aligned}
$$

joining all the roots of its argument to a newly created root. Clearly, $B_{+}$reminds of an operad multiplication. It is easily seen that $B_{+}$is not exact and therefore a generator (among others) of $\operatorname{HH}_{\epsilon}^{1}\left(\mathcal{H}_{r t}\right)$. Foissy [23] showed that $L \mapsto L(\mathbb{I})$ is an onto map $\operatorname{HH}_{\epsilon}^{1}\left(\mathcal{H}_{r t}\right) \rightarrow$ Prim $\mathcal{H}_{r t}$. The higher Hochschild cohomology $(n \geq 2)$ of $\mathcal{H}_{r t}$ is known to vanish 23. The pair $\left(\mathcal{H}_{r t}, B_{+}\right)$is the universal model for all Hopf algebras of Feynman graphs and their 1-cocycles [14. Let us now turn to those 1-cocycles of $\mathcal{H}$. Clearly, every primitve graph $\gamma$ gives rise to a 1-cocycle $B_{+}^{\gamma}$ defined as the operator which inserts its argument, a product of graphs, into $\gamma$ in all possible ways. Here is a simple example:

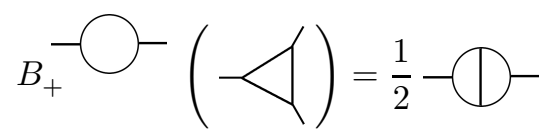

See [30] for the general definition involving some combinatorics of insertion places and symmetries.

It is an important consequence of the $B_{+}^{\gamma}$ satisfying the cocycle condition (5) that

$$
\left(S_{R}^{\phi} * \phi\right) B_{+}=(1-R) \tilde{B}_{+}\left(S_{R}^{\phi} * \phi\right)
$$

where $\tilde{B}_{+}$is the push-forward of $B_{+}$along the Feynman rules $\phi$. In other words, $\tilde{B}_{+}^{\gamma}$ is the integral operator corresponding to the skeleton graph $\gamma$. This is the combinatorial key to the proof of locality of counterterms and finiteness of renormalization [13, 28, 2, 3. Indeed, equation (6) says that after treating all subdivergences, an overall subtraction $(1-R)$ suffices. The only analytic ingredient is 
Weinberg's theorem applied to the primitive graphs. In [2] it is emphasized that $\mathcal{H}$ is actually generated (and determined) by the action of prescribed 1-cocycles and the multiplication. A version of (6) with decorated trees is available which describes renormalization in coordinate space [2].

The 1-cocycles $B_{+}^{\gamma}$ give rise to a number of useful Hopf subalgebras of $\mathcal{H}$. Many of them are isomorphic. They are studied in [3] on the model of decorated rooted trees, and we will come back to them in the next section. In 30] one of the authors showed that in nonabelian gauge theories, the existence of a certain Hopf subalgebra, generated by 1-cocycles, is closely related to the SlavnovTaylor identities for the couplings to hold. In a similar spirit, van Suijlekom showed that, in QED, Ward-Takahashi identities, and in nonabelian Yang-Mills theories, the Slavnov-Taylor identities for the couplings generate Hopf ideals $\mathcal{I}$ of $\mathcal{H}$ such that the quotients $\mathcal{H} / \mathcal{I}$ are defined and the Feynman rules factor through them [37, 38. The Hopf algebra $\mathcal{H}$ for QED had been studied before in $11,33,39$.

\section{Dyson-Schwinger equations}

The ultimate application of the Hochschild 1-cocycles introduced in the previous section aims at non-perturbative results. Dyson-Schwinger equations, reorganized using the correspondence $\operatorname{Prim} \mathcal{H} \rightarrow \mathrm{HH}_{\epsilon}^{1}(\mathcal{H})$, become recursive equations in $\mathcal{H}[[\alpha]], \alpha$ the coupling constant, with contributions from (degree 1) 1-cocycles. The Feynman rules connect them to the usual integral kernel representation. We remain in the massless $\phi^{3}$ theory in 6 dimensions for the moment. Let $\Gamma^{\prec}$ be the full 1PI vertex function,

$$
\Gamma^{\prec}=\mathbb{I}+\sum_{\operatorname{res} \Gamma=\prec} \alpha^{|\Gamma|} \frac{\Gamma}{\operatorname{Sym} \Gamma}
$$

(normalized such that the tree level contribution equals 1). This is a formal power series in $\alpha$ with values in $\mathcal{H}$. Here $\operatorname{res} \Gamma$ is the result of collapsing all internal lines of $\Gamma$. The graph res $\Gamma$ is called the residue of $\Gamma$. In a renormalizable theory, res can be seen as a map from the set of generators of $\mathcal{H}$ to the terms in the Lagrangian. For instance, in the $\phi^{3}$ theory, vertex graphs have residue $\prec$, and self energy graphs have residue - . The number Sym $\Gamma$ denotes the order of the group of automorphisms of $\Gamma$, defined in detail for example in [30, 38]. Similarly, the full inverse propagator $\Gamma^{-}$is represented by

$$
\Gamma^{-}=\mathbb{I}-\sum_{\operatorname{res} \Gamma=-} \alpha^{|\Gamma|} \frac{\Gamma}{\operatorname{Sym} \Gamma} .
$$

These series can be reorganized by summing only over primitive graphs, with all possible insertions into these primitive graphs. In $\mathcal{H}$, the insertions are afforded 
by the corresponding Hochschild 1-cocycles. Indeed,

$$
\begin{aligned}
\Gamma^{\prec} & =\mathbb{I}+\sum_{\gamma \in \operatorname{Prim} \mathcal{H}, \text { res } \gamma=\alpha} \frac{\alpha^{|\gamma|} B_{+}^{\gamma}\left(\Gamma^{\prec} Q^{|\gamma|}\right)}{\operatorname{Sym} \gamma} \\
\Gamma^{-} & =\mathbb{I}-\sum_{\gamma \in \operatorname{Prim} \mathcal{H}, \operatorname{res} \gamma=-} \frac{\alpha^{|\gamma|} B_{+}^{\gamma}\left(\Gamma^{-} Q^{|\gamma|}\right)}{\operatorname{Sym} \gamma} .
\end{aligned}
$$

The universal invariant charge $Q$ is a monomial in the $\Gamma^{r}$ and their inverses, where $r$ are residues (terms in the Lagrangian) provided by the theory. In $\phi^{3}$ theory we have $Q=\left(\Gamma^{\prec}\right)^{2}\left(\Gamma^{-}\right)^{-3}$. In $\phi^{3}$ theory, the universality of $Q$ (i. e. the fact that the same $Q$ is good for all Dyson-Schwinger equations of the theory) comes from a simple topological argument. In nonabelian gauge theories however, the universality of $Q$ takes care that the solution of the corresponding system of coupled Dyson-Schwinger equations gives rise to a Hopf subalgebra and therefore amounts to the Slavnov-Taylor identities for the couplings 30.

The system (9) of coupled Dyson-Schwinger equations has (718) as its solution. Note that in the first equation of (9) an infinite number of cocycles contributes as there are infinitely many primitive vertex graphs in $\phi_{6}^{3}$ theory - the second equation has only finitely many contributions - here one. Before we describe how to actually attempt to solve equations of this kind analytically (application of the Feynman rules $\phi$ ), we discuss the combinatorial ramifications of this construction in the Hopf algebra. It makes sense to call all (systems of) recursive equations of the form

$$
\begin{aligned}
X_{1} & =\mathbb{I} \pm \sum_{n} \alpha^{k_{n}^{1}} B_{+}^{d_{n}^{1}}\left(M_{n}^{1}\right) \\
& \cdots \\
X_{s} & =\mathbb{I} \pm \sum_{n} \alpha^{k_{n}^{s}} B_{+}^{d_{n}^{s}}\left(M_{n}^{s}\right)
\end{aligned}
$$

combinatorial Dyson-Schwinger equations, and to study their combinatorics. Here, the $B_{+}^{d_{n}}$ are non-exact Hochschild 1-cocycles and the $M_{n}$ are monomials in the $X_{1} \ldots X_{s}$. In [3] we studied a large class of single (uncoupled) combinatorial Dyson-Schwinger equations in a decorated version of $\mathcal{H}_{r t}$ as a model for vertex insertions:

$$
X=\mathbb{I}+\sum_{n=1}^{\infty} \alpha^{n} w_{n} B_{+}^{d_{n}}\left(X^{n+1}\right)
$$

where the $w_{n} \in \mathbb{Q}$. For example, $X=\mathbb{I}+\alpha B_{+}\left(X^{2}\right)+\alpha^{2} B_{+}\left(X^{3}\right)$ is in this class. It turns out [28, 3] that the coefficients $c_{n}$ of $X$, defined by $X=\sum_{n=0}^{\infty} \alpha^{n} c_{n}$ generate a Hopf subalgebra themselves:

$$
\Delta\left(c_{n}\right)=\sum_{k=0}^{n} P_{k}^{n} \otimes c_{k} .
$$


The $P_{k}^{n}$ are homogeneous polynomials of degree $n-k$ in the $c_{l}, l \leq n$. These polynomials have been worked out explicitly in [3]. One notices in particular that the $P_{k}^{n}$ are independent of the $w_{n}$ and $B_{+}^{d_{n}}$, and hence that under mild assumptions (on the algebraic independence of the $c_{n}$ ) the Hopf subalgebras generated this way are actually isomorphic. For example, $X=\mathbb{I}+\alpha B_{+}\left(X^{2}\right)+\alpha^{2} B_{+}\left(X^{3}\right)$ and $X=\mathbb{I}+\alpha B_{+}\left(X^{2}\right)$ yield isomorphic Hopf subalgebras. This is an aspect of the fact that truncation of Dyson-Schwinger equations - considering only a finite instead of an infinite number of contributing cocycles - does make (at least combinatorial) sense. Indeed, the combinatorics remain invariant. Similar results hold for Dyson-Schwinger equations in the true Hopf algebra of graphs $\mathcal{H}$ where things are a bit more difficult though as the cocycles there involve some bookkeeping of insertion places.

The simplest nontrivial Dyson-Schwinger equation one can think of is the linear one:

$$
X=\mathbb{I}+\alpha B_{+}(X) .
$$

Its solution is given by $X=\sum_{n=0}^{\infty} \alpha^{n}\left(B_{+}\right)^{n}(\mathbb{I})$. In this case $X$ is grouplike and the corresponding Hopf subalgebra of $c_{n} \mathrm{~s}$ is cocommutative [25. A typical and important non-linear Dyson-Schwinger equation arises from propagator insertions:

$$
X=\mathbb{I}-\alpha B_{+}(1 / X),
$$

for example the massless fermion propagator in Yukawa theory where only the fermion line obtains radiative corrections (other corrections are ignored). This problem has been studied and solved by Broadhurst and one of the authors in [12] and revisited recently by one of the authors and Yeats [35]. As we now turn to the analytic aspects of Dyson-Schwinger equations, we briefly sketch the general approach presented in [35] on how to successfully treat the nonlinearity of Dyson-Schwinger equations. Indeed, the linear Dyson-Schwinger equations can be solved by a simple scaling ansatz [25]. In any case, let $\gamma$ be a primitive graph. The following works for amplitudes which depend on a single scale, so let us assume a massless situation with only one non-zero external momentum how more than one external momentum (vertex insertions) are incorporated by enlarging the set of primitive elements is sketched in 32. The grafting operator $B_{+}^{\gamma}$ associated to $\gamma$ translates to an integral operator under the (renormalized) Feynman rules

$$
\phi_{R}\left(B_{+}^{\gamma}\right)(\mathbb{I})\left(p^{2} / \mu^{2}\right)=\int\left(I_{\gamma}(k, p)-I_{\gamma}(k, \mu)\right) d k
$$

where $I_{\gamma}$ is the integral kernel corresponding to $\gamma$, the internal momenta are denoted by $k$, the external momentum by $p$, and $\mu$ is the fixed momentum at which we subtract: $R(x)=\left.x\right|_{p^{2}=\mu^{2}}$.

In the following we stick to the special case discussed in 35 where only one internal edge is allowed to receive corrections. The integral kernel $\phi\left(B_{+}^{\gamma}\right)$ defines 
a Mellin transform

$$
F(\rho)=\int I_{\gamma}(k, \mu)\left(k_{i}^{2}\right)^{-\rho} d k
$$

where $k_{i}$ is the momentum of the internal edge of $\gamma$ at which insertions may take place (here the fermion line). If there are several insertion sites, obvious multiple Mellin transforms become necessary. The case of two (propagator) insertion places has been studied, at the same example, in [35].

The function $F(\rho)$ has a simple pole in $\rho$ at 0 . We write

$$
F(\rho)=\frac{r}{\rho}+\sum_{n=0}^{\infty} f_{n} \rho^{n}
$$

We denote $L=\log p^{2} / \mu^{2}$. Clearly $\phi_{R}(X)=1+\sum_{n} \gamma_{n} L^{n}$. An important result of 35 is that, even in the difficult nonlinear situation, the anomalous dimension $\gamma_{1}$ is implicitly defined by the residue $r$ and Taylor coefficients $f_{n}$ of the Mellin transform $F$. On the other hand, all the $\gamma_{n}$ for $n \geq 2$, are recursively defined in terms of the $\gamma_{i}, i<n$. This last statement amounts to a renormalization group argument that is afforded in the Hopf algebra by the scattering formula of 16. Curiously, for this argument only a linearized part of the coproduct is needed. We refer to 35 for the actual algorithm. For a linear Dyson-Schwinger equation, the situation is considerably simpler as the $\gamma_{n}=0$ for $n \geq 2$ since $X$ is grouplike [25].

Let us restate the results for the high energy sector of non-linear Dyson-Schwinger equations [12, 35]: Primitive graphs $\gamma$ define Mellin transforms via their integral kernels $\tilde{B}_{+}^{\gamma}$. The anomalous dimension $\gamma_{1}$ is implicitly determined order by order from the coefficients of those Mellin transforms. All non-leading log coefficients $\gamma_{n}$ are recursively determined by $\gamma_{1}$, thanks to the renormalization group. This reduces, in principle, the problem to a study of all the primitive graphs and the intricacies of insertion places.

Finding useful representations of those Mellin transforms - even one-dimensional ones - of higher loop order skeleton graphs is difficult. However, the two-loop primitive vertex in massless Yukawa theory has been worked out by Bierenbaum, Weinzierl and one of the authors in [4, a result that can be applied to other theories as well. Combined with the algebraic treatment [12, 3, 35] sketched in the previous paragraphs and new geometric insight on primitive graphs (see section (5), there is reasonable hope that actual solutions of Dyson-Schwinger equations will be more accessible in the future.

Using the Dyson-Schwinger analysis, one of the authors and Yeats 34 were able to deduce a bound for the convergence of superficially divergent amplitudes/structure functions from the (desirable) existence of a bound for the superficially convergent amplitudes. 


\section{Feynman integrals and periods of mixed (Tate) Hodge structures}

A primitive graph $\Gamma \in$ Prim $\mathcal{H}$ defines a real number $r_{\Gamma}$, called the residue of $\Gamma$, which is independent of the renormalization scheme. In the case that $\Gamma$ is massless and has one external momentum $p$, the residue $r_{\Gamma}$ is the coefficient of $\log p^{2} / \mu^{2}$ in $\phi_{R}(\Gamma)=(1-R) \phi(\Gamma)$. It coincides with the coefficient $r$ of the Mellin transform introduced in the previous section. One may ask what kind of a number $r$ is, for example if it is rational or algebraic. The origin of this question is that the irrational or transcendental numbers that show up for various $\Gamma$ strongly suggest a motivic interpretation of the $r_{\Gamma}$. Indeed, explicit calculations $9,10,8$, display patterns of Riemann zeta and multiple zeta values that are known to be periods of mixed Tate Hodge structures - here the periods are provided by the Feynman rules which produce $\Gamma \mapsto r_{\Gamma}$. By disproving a related conjecture of Kontsevich, Belkale and Brosnan [1] have shown that not all these Feynman motives must be mixed Tate, so one may expect a larger class of Feynman periods than multiple zeta values. Our detailed understanding of these phenomena is still far from complete, and only some very first steps have been made in the last few years. However, techniques developed in recent work by Bloch, Esnault and one of the authors 7 do permit reasonable insight for some special cases which we briefly sketch in the following.

Let $\Gamma$ be a logarithmically divergent massless primitive graph with one external momentum $p$. It is convenient to work in the "Schwinger" parametric representation 24] obtained by the usual trick of replacing propagators

$$
\frac{1}{k^{2}}=\int_{0}^{\infty} d a e^{-a k^{2}}
$$

and performing the loop integrations (Gaussian integrals) first which leaves us with a (divergent) integral over various Schwinger parameters $a$. It is a classical exercise [24, 7, 6] to show that in four dimensions, up to some powers of $i$ and $2 \pi$

$$
\phi(\Gamma)=\int_{0}^{\infty} d a_{1} \ldots d a_{n} \frac{e^{-Q_{\Gamma}\left(a, p^{2}\right) / \Psi_{\Gamma}(a)}}{\Psi_{\Gamma}^{2}(a)}
$$

where $n$ is the number of edges of $\Gamma . Q_{\Gamma}$ and $\Psi_{\Gamma}$ are graph polynomials of $\Gamma$, where $\Psi_{\Gamma}$, sometimes called Symanzik or Kirchhoff polynomial, is defined as follows: Let $T(\Gamma)$ be the set of spanning trees of $\Gamma$, i. e. the set of connected simply connected subgraphs which meet all vertices of $\Gamma$. We think of the edges $e$ of $\Gamma$ as being numbered from 1 to $n$. Then

$$
\Psi_{\Gamma}=\sum_{t \in T(\Gamma)} \prod_{e \notin t} a_{e}
$$


This is a homogeneous polynomial in the $a_{i}$ of degree $\left|H_{1}(\Gamma)\right|$. It is easily seen (scaling behaviour of $Q_{\Gamma}$ and $\Psi_{\Gamma}$ ) that $r_{\Gamma}=\frac{\partial \phi_{R}(\Gamma)}{\partial \log p^{2} / \mu^{2}}$ is extracted from $\phi(\Gamma)$ by considering the $a_{i}$ as homogeneous coordinates of $\mathbb{P}^{n-1}(\mathbb{R})$ and evaluating at $p^{2}=0$ :

$$
r_{\Gamma}=\int_{\sigma \subset \mathbb{P}^{n-1}(\mathbb{R})} \frac{\Omega}{\Psi_{\Gamma}^{2}}
$$

where $\sigma=\left\{\left[a_{1}, \ldots, a_{n}\right]\right.$ : all $a_{i}$ can be choosen $\left.\geq 0\right\}$ and $\Omega$ is a volume form on $\mathbb{P}^{n-1}$. Let $X_{\Gamma}:=\left\{\Psi_{\Gamma}=0\right\} \subset \mathbb{P}^{n-1}$. If $\left|H_{1}(\Gamma)\right|=1$, the integrand in (10) has no poles. If $\left|H_{1}(\Gamma)\right|>1$, poles will show up on the union $\Delta=\bigcup_{\gamma \subsetneq \Gamma, H_{1}(\gamma) \neq 0} L_{\gamma}$ of coordinate linear spaces $L_{\gamma}=\left\{a_{e}=0\right.$ for $e$ edge of $\left.\gamma\right\}$ - these need to be separated from the chain of integration by blowing up. The blowups being understood, the Feynman motive is, by abuse of notation,

$$
H^{n-1}\left(\mathbb{P}^{n-1}-X_{\Gamma}, \Delta-\Delta \cap X_{\Gamma}\right)
$$

with Feynman period given by (10). See [7, 6] for details. Some particularly accessible examples are the wheel with $n$ spokes graphs

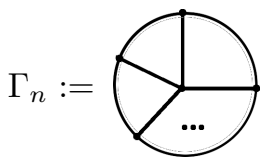

studied extensively in [7. The corresponding Feynman periods (10) yield rational multiples of zeta values [9]

$$
r_{\Gamma_{n}} \in \zeta(2 n-3) \mathbb{Q} .
$$

Due to the simple topology of the $\Gamma_{n}$, the geometry of the pairs $\left(X_{\Gamma_{n}}, \Delta_{\Gamma_{n}}\right)$ are well understood and the corresponding motives have been worked out explicitly [7. The methods used are however nontrivial and not immediately applicable to more general situations.

When confronted with non-primitive graphs, i. e. graphs with subdivergences, there are more than one period to consider. In the Schwinger parameter picture, subdivergences arise when poles appear along exceptional divisors as pieces of $\Delta$ are blown up. This situation can be understood using limiting mixed Hodge structures [6], see also [31, 36] for a toy model approach to the combinatorics involved. In 6 it is also shown how the Hopf algebra $\mathcal{H}$ of graphs lifts to the category of motives. For the motivic role of solutions of Dyson-Schwinger equations we refer to work in progress. Finally we mention that there is related work by Connes and Marcolli [18, 19] who attack the problem via Riemann-Hilbert correspondences and motivic Galois theory.

Acknowledgements. We thank Spencer Bloch and Karen Yeats for discussion on the subject of this review. The first named author (C. B.) thanks the organizers of the ICMP 2006 and the IHES for general support. His research is 
supported by the Deutsche Forschungsgemeinschaft. The IHES, Boston University and the Erwin-Schrödinger-Institute are gratefully acknowledged for their kind hospitality. At the time of writing this article, C. B. is visiting the ESI as a Junior Research Fellow.

\section{References}

[1] P. Belkale and P. Brosnan. Matroids, motives, and a conjecture of Kontsevich. Duke Math. J. 116, (1):147-188, 2003. math.AG/0012198.

[2] C. Bergbauer and D. Kreimer. The Hopf algebra of rooted trees in EpsteinGlaser renormalization. Ann. Henri Poincare, 6:343-367, 2004. hepth/0403207.

[3] C. Bergbauer and D. Kreimer. Hopf algebras in renormalization theory: Locality and Dyson-Schwinger equations from Hochschild cohomology. IRMA Lect. Math. Theor. Phys., 10:133-164, 2006. hep-th/0506190.

[4] I. Bierenbaum, D. Kreimer, and S. Weinzierl. The next-to-ladder approximation for Dyson-Schwinger equations. Phys. Lett. , B646:129-133, 2007. hep-th/0612180.

[5] S. Bloch. Mixed Hodge structures and motives in physics. Talk at the conference on Motives and Algebraic Cycles, Fields Institute, March 2007.

[6] S. Bloch. Motives Associated to Graphs. Takagi Lectures, Kyoto, November 2006. Available online at http://www.math.uchicago.edu/ bloch/.

[7] S. Bloch, H. Esnault, and D. Kreimer. On Motives Associated to Graph Polynomials. Commun. Math. Phys., 267:181-225, 2006. math.AG/0510011.

[8] D. Broadhurst, J. Gracey, and D. Kreimer. Beyond the triangle and uniqueness relations: Non-zeta counterterms at large $\mathrm{N}$ from positive knots. Z. Phys., C75:559-574, 1997. hep-th/9607174.

[9] D. Broadhurst and D. Kreimer. Knots and numbers in $\Phi^{4}$ theory to 7 loops and beyond. Int. J. Mod. Phys., C6:519-524, 1995. hep-ph/9504352.

[10] D. Broadhurst and D. Kreimer. Association of multiple zeta values with positive knots via feynman diagrams up to 9 loops. Phys. Lett. , B393:403412, 1997. hep-th/9609128.

[11] D. Broadhurst and D. Kreimer. Renormalization automated by Hopf algebra. J. Symb. Comput., 27:581, 1999. hep-th/9810087.

[12] D. Broadhurst and D. Kreimer. Exact solutions of Dyson-Schwinger equations for iterated one-loop integrals and propagator-coupling duality. Nucl. Phys. , B600:403-422, 2001. hep-th/0012146. 
[13] J. Collins. Renormalization. Cambridge Monographs on Mathematical Physics. Cambridge University Press, 1984.

[14] A. Connes and D. Kreimer. Hopf algebras, renormalization and noncommutative geometry. Commun. Math. Phys., 199:203-242, 1998. hepth/9808042.

[15] A. Connes and D. Kreimer. Renormalization in quantum field theory and the Riemann- Hilbert problem I: The Hopf algebra structure of graphs and the main theorem. Comm. Math. Phys., 210:249-273, 2000. hepth/9912092.

[16] A. Connes and D. Kreimer. Renormalization in quantum field theory and the Riemann-Hilbert problem II: The beta-function, diffeomorphisms and the renormalization group. Commun. Math. Phys., 216:215-241, 2001. hepth/0003188.

[17] A. Connes and D. Kreimer. Insertion and elimination: The doubly infinite Lie algebra of Feynman graphs. Ann. Henri Poincaré, 3:411-433, 2002. hep-th/0201157.

[18] A. Connes and M. Marcolli. From Physics to Number Theory via Noncommutative Geometry, Part II: Renormalization, the Riemann-Hilbert correspondence, and motivic Galois theory. In Frontiers in Number Theory, Physics, and Geometry II, pages 617-713. Springer, 2006. hep-th/0411114.

[19] A. Connes and M. Marcolli. Quantum fields and motives. J. Geom. Phys., 56(1):55-85, 2006. hep-th/0504085.

[20] K. Ebrahimi-Fard, L. Guo, and D. Kreimer. Integrable renormalization I: The ladder case. J. Math. Phys., 45:3758-3769, 2004. hep-th/0402095.

[21] K. Ebrahimi-Fard, L. Guo, and D. Kreimer. Spitzer's identity and the algebraic Birkhoff decomposition in pQFT. J. Phys., A37:11037-11052, 2004. hep-th/0407082.

[22] K. Ebrahimi-Fard, L. Guo, and D. Kreimer. Integrable renormalization II: The general case. Ann. Henri Poincaré, 6:369-395, 2005. hep-th/0403118.

[23] L. Foissy. Les algèbres de Hopf des arbres enracinés I-II. Bull. Sci. Math., 126:193-239 and 249-288, 2002.

[24] C. Itzykson and J. -B. Zuber. Quantum field theory. McGraw-Hill, 1980.

[25] D. Kreimer. Étude for linear Dyson-Schwinger equations. IHES Preprint $\mathrm{P} / 06 / 23$.

[26] D. Kreimer. On the Hopf algebra structure of perturbative quantum field theories. Adv. Theor. Math. Phys., 2:303-334, 1998. q-alg/9707029. 
[27] D. Kreimer. On overlapping divergences. Commun. Math. Phys., 204:669, 1999. hep-th/9810022.

[28] D. Kreimer. Factorization in quantum field theory: An exercise in Hopf algebras and local singularities. 2003. Contributed to Les Houches School of Physics: Frontiers in Number Theory, Physics and Geometry, Les Houches, France, 9-21 Mar 2003. hep-th/0306020.

[29] D. Kreimer. What is the trouble with Dyson-Schwinger equations? Nucl. Phys. Proc. Suppl., 135:238-242, 2004. hep-th/0407016.

[30] D. Kreimer. Anatomy of a gauge theory. Annals Phys. , 321:2757-2781, 2006.

[31] D. Kreimer. The residues of quantum field theory: Numbers we should know. In C. Consani and M. Marcolli, editors, Noncommutative Geometry and Number Theory (Bonn, 2003), pages 187-204. Vieweg, 2006. hepth/0404090.

[32] D. Kreimer. Dyson Schwinger equations: From Hopf algebras to number theory. In I. Binder and D. Kreimer, editors, Universality and Renormalization, volume 50 of Fields Inst. Comm., pages 225-248. AMS, 2007. hep-th/0609004.

[33] D. Kreimer and R. Delbourgo. Using the Hopf algebra structure of QFT in calculations. Phys. Rev. , D60:105025, 1999. hep-th/9903249.

[34] D. Kreimer and K. Yeats. Recursion and growth estimates in renormalizable quantum field theory. hep-th/0612179.

[35] D. Kreimer and K. Yeats. An etude in non-linear Dyson-Schwinger equations. Nucl. Phys. Proc. Suppl. , 160:116-121, 2006. hep-th/0605096.

[36] I. Mencattini and D. Kreimer. The structure of the Ladder InsertionElimination Lie algebra. Commun. Math. Phys., 259:413-432, 2005. math$\mathrm{ph} / 0408053$.

[37] W. van Suijlekom. The Hopf algebra of Feynman graphs in QED. Lett. Math. Phys. , 77:265-281, 2006. hep-th/0602126.

[38] W. van Suijlekom. Renormalization of gauge fields: A Hopf algebra approach. 2006. hep-th/0610137.

[39] D. Volovich, I. and Prokhorenko. Renormalizations in quantum electrodynamics, and Hopf algebras. Tr. Mat. Inst. Steklova, 245(Izbr. Vopr. padich. Mat. Fiz. i Anal. ):288-295, 2004. 